Version: 9 June 2018

\title{
The formation of cluster elliptical galaxies as revealed by extensive star formation
}

\author{
J. A. Stevens*, R. J. Ivison*, J. S. Dunlop†, Ian Smail $\ddagger$ W. J. Percival†, D. H. Hughes§ \\ H. J. A. Röttgering\|, W. J. M. van Breugel \& \& M. Reuland \\ * Astronomy Technology Centre, Royal Observatory, Blackford Hill, Edinburgh \\ EH9 3HJ, UK \\ $\dagger$ Institute for Astronomy, University of Edinburgh, Blackford Hill, \\ Edinburgh EH9 3HJ \\ $\ddagger$ Institute for Computational Cosmology, University of Durham, \\ South Road, Durham DH1 3LE \\ $\S$ Instituto Nacional de Astrofisica, Optica y Electronica, Apartado Postal 51 y 216, 72000 \\ Puebla, Mexico \\ || Leiden Observatory, PO Box 9513, 2300 Leiden, The Netherlands \\ I Institute of Geophysics and Planetary Physics, Lawrence Livermore National Laboratory, \\ PO Box 808, Livermore, CA 94459, USA
}

The most massive galaxies in the present-day Universe are found to lie in the centres of rich clusters. They have old, coeval stellar populations suggesting that the bulk of their stars must have formed at early epochs in spectacular starbursts $^{1}$ - luminous phenomena at submillimetre wavelengths ${ }^{2}$. The most popular model of galaxy formation predicts that these galaxies form in protoclusters at high-density peaks in the early Universe ${ }^{3}$. Such peaks are signposted by massive high-redshift radio galaxies ${ }^{4}$. Here we report deep submillimetre mapping of seven high-redshift radio galaxies and their environments. These data confirm not only the presence of spatially extended massive star-formation activity in the radio galaxies themselves, but also in companion objects previously undetected at any wavelength. The prevalence, orientation, and inferred masses of these submillimetre companion galaxies suggest that we are witnessing the synchronous formation of the most luminous elliptical galaxies found today at the centres of rich galaxy clusters. 
Whilst existing submillimetre studies of high-redshift radio galaxies (hereafter HzRGs) have shown that their star-formation rates are large enough to build a massive galaxy in $<1 \mathrm{Gyr}^{2,5,6,7}$ they have provided no information on the spatial extent of this emission or on the star-formation activity in their environments. We have therefore mapped a sample of seven objects with redshifts ranging from 2.2 to 4.3 at a wavelength of $850 \mu \mathrm{m}$ with the Submillimetre Common-User Bolometer Array (SCUBA) ${ }^{8}$ on the James Clerk Maxwell Telescope (JCMT). The targets were chosen from those sources found to be submillimetre bright in the previous SCUBA surveys of HzRGs ${ }^{6,7}$. Our new maps illustrate the distribution of dust-reradiated emission in and around the HzRGs on scales from $5^{\prime \prime}$ to $160^{\prime \prime}$, or $30 \mathrm{kpc}$ to $1 \mathrm{Mpc}$. We illustrate the seven submillimetre maps from this survey in Figure 1; the orientation of the radio jets of each HzRG is represented by tick marks on these maps.

One of the most striking aspects of the submillimetre maps is that the dust emission from the central radio galaxy is resolved in at least five of the seven sources - even with the coarse beam of the JCMT. In Figure 2 and Table 1 we present simple two-dimensional Gaussian fits to the data which, while not giving a true reflection of the physical situation, at least provide a quantitative measure of the spatial extent of the dust emission. This emission is sometimes in the form of several partially-resolved or merged clumps (typified by 8C 1909+722), sometimes in an apparently smoother distribution (e.g. 4C 60.07), and is more extended than the radio emission in most cases. The extent of the dust emission ranges from 50 to $250 \mathrm{kpc}$, a physically interesting size because (1) the corresponding half-light radii $(30-150 \mathrm{kpc})$ are equivalent to those of brightest cluster galaxies in the local Universe ${ }^{9}$, and (2) gas-dynamical simulations of major galaxy mergers predict that star formation should peak when the galaxies are separated on approximately this scale. ${ }^{10}$ Higher resolution, high signal-to-noise millimetre/submillimetre imaging observations are required to investigate these possibilities.

A second striking feature of our SCUBA maps is that several of the fields also contain serendipitous detections of new, luminous submillimetre galaxies. In Table 1 we list the properties of those companion galaxies with peak signal-to-noise ratio $>4$. Note that several of these companions are, like the HzRGs, resolved at $850 \mu \mathrm{m}$. The source density of companions is also higher than found in 'blank-field' surveys; current submillimetre number counts ${ }^{11}$ indicate that we should find, on average, about one random submillimetre source per SCUBA field at a flux level of $S_{850}>5-6$ mJy compared to our detection of approximately twice this number (Table 1). Furthermore, the 4C 41.17 and 8C 1909+722 fields each contain a companion source with $S_{850}>20 \mathrm{mJy}$. By comparison, the biggest blank field survey conducted to date ${ }^{12}$, which has an area $\sim 7$ times that of our own, contains no robust sources with $\mathrm{S}_{850}>15 \mathrm{mJy}$.

Arguably the most surprising feature of the maps is the observed aligments between the HzRG radio axis and (1) the submillimetre emission (2) the brightest submillimetre 'companions'. The significance of these alignments is presented and quantified in Figure 3. These results can be interpreted as follows. First, while one might be tempted to conclude that the first alignment effect is indicative of jet-induced star formation similar to the radio-UV alignment effect previously reported in $\mathrm{HzRGs}^{13,14}$, the fact that in all but two cases, the radio source is also aligned with submillimetre source on scales well beyond the 
size of the radio emission indicates that this is unlikely to be the complete explanation. Moreover, this model clearly cannot explain why the submillimetre emission from the HzRGs appears to be aligned with positions of the brightest companion sources in their vicinity (in at least 4 out of 7 cases).

There is one model that can explain both alignment effects. We propose that the brightest submillimetre companions trace the large-scale structure around the HzRG and that these directions thus contain the densest cross-sections of gas. Next, by selecting some of the very brightest known radio sources at this epoch we will then have (inadvertently, but probably inevitably) selected sources which happen to have produced jets aligned with the densest regions of gas, thereby producing very effective working surfaces and the brightest hot spots. Such a selection effect has been suggested before to explain apparent large-scale optical-radio alignment effects at lower redshift. ${ }^{15,4}$

Of course, an important corollary to this interpretation is that the brightest companion sources seen in the submillimetre maps must lie at the same redshift as the radio galaxies, occupying the same large-scale structure. However, the fact that extension of this analysis to the second brightest apparent companion object does not reveal a significant alignment effect shows that not all of the objects seen in these images need lie at the same redshift as, and be physically associated with the radio galaxies. This is consistent with our earlier estimate of the rate of contamination in these fields by unrelated submillimetre sources. Thus the analysis of these possible alignment effects indicates that the submillimetre emission from the radio galaxies themselves, and that of the brightest companions, is tracing the large scale structure around radio galaxies at $z \simeq 3$. We note that for $53 \mathrm{~W} 002$, a HzRG not included in our sample, it has been shown with optical spectroscopy that the brightest submillimetre companion is indeed at the same redshift as the radio galaxy. ${ }^{16} \mathrm{~A}$ similar conclusion can be inferred for PKS 1138 - 262 where the brightest submillimetre companion is coincident with one of 5 actuve galactic nuclei (AGN) forming a radio-aligned filament in the plane of the sky, and which has the same redshift as the HzRG based on narrow-band imaging of redshifted Ly $\alpha$ emission. ${ }^{17}$

Since we have shown that these fields contain overdensities of submillimetre sources, it is of interest to ask what is the typical mass of these galaxies. We can do this by assuming that they lie at the same redshift as the HzRGs. The dust masses of the HzRGs, calculated from their $850-\mu \mathrm{m}$ fluxes $^{18}$ are given in Table 1 . Using standard assumptions we can convert these dust masses into total gas masses, and hence estimate the masses of their associated dark matter haloes (see table caption for details). We estimate that they reside in dark matter halos with masses in excess of $10^{12} \mathrm{M}_{\odot}$. Since the submillimetre flux densities of the companions are similar to those of the HzRGs we can infer that they also reside in dark matter halos with masses in excess of $10^{12} \mathrm{M}_{\odot}$. We therefore conclude that these regions can contain more than one very massive and gas-rich galaxy of $>10^{12} \mathrm{M}_{\odot}$. Can we reconcile this result with the hierarchical models of galaxy and structure formation?

By design we have selected highly-biased regions of the high-redshift universe by imaging around some of the most luminous radio galaxies at these epochs, which are expected to host some of the most massive black holes. We can attempt to quantify the impact of this bias by exploring the predictions of numerical simulations of the collapse of Cold Dark Matter haloes within a $\Lambda$-dominated universe. The details and results of these sim- 
ulations are given in Figure 4. These simulations do indeed appear to be in accord with the observations, i.e. both data and theory suggest that the submillimetre companions revealed by our SCUBA imaging are associated with dark-matter halos more massive than $10^{12} \mathrm{M}_{\odot}$. This conclusion is, however, subject to an assumption about the duty cycles of the submillimetre luminous phase; if true it would imply that the vast majority of such massive halos are actively forming stars at the epochs sampled by these images. This is not unreasonable, especially since we have deliberately targetted fields in which at least one massive object (i.e. the HzRG) is actively engaged in intense star formation.

Pursuing this comparison one step further, we can explore the predicted and observed distribution of companion objects in this high-mass range. In fact, while the average number of such companions in the simulated SCUBA images centred on the HzRG is 1, this average arises from a skewed distribution. As shown in Figure 4, of the 40 high-mass haloes investigated, $50 \%$ have no such massive companions, with the average of 1 resulting from the fact that, of the remaining half, $40 \%$ have 2 companions or more. We have over-plotted in Figure 4 the corresponding histogram of companion incidence for the 7 HzRGs imaged in this study (after statistically correcting for field contamination). This comparison at least illustrates that the distribution of companion incidence in our data is consistent with the prediction of the simulations, i.e. typically we see either no companion, or $\geq 2$ companions.

The average number of companions, the distribution of companion incidence, and the inferred baryonic gas masses of the companions are all most consistent with the interpretation that the SCUBA sources uncovered in this study are the progenitors of massive present-day cluster ellipticals. In this case, since elliptical galaxies are known to contain massive black holes with mass proportional to that of the spheroid, ${ }^{19}$ it is reasonable to assume that the black hole and stellar mass grow coevally from the same gas reservoir. This in turn suggests that the companion objects should contain buried AGN. ${ }^{20,21}$ First results show a high rate of correspondence between the submillimetre companions and luminous X-ray sources, ${ }^{16,22}$ suggesting that this is indeed the case. We are presently pursuing highresolution follow-up observations of these fields at millimetre and optical/near-infrared wavelengths. The former will yield information on source structure while the latter will reveal counterparts for spectroscopic redshift determination on 10-m class telescopes. 


\section{References.}

1. Ellis, R. S. et al. The homogeneity of spheroidal populations in distant clusters. Astrophys. J. 483, 582-596 (1997).

2. Dunlop, J. S. et al. Detection of a large mass of dust in a radio galaxy at redshift $z=3.8$. Nature 370, 347-349 (1994).

3. Kauffmann, G. The age of elliptical galaxies and bulges in a merger model. Mon. Not. R. Astron. Soc. 281, 487-492 (1996).

4. West M. J. Anisotropic mergers at high redshifts: the formation of $\mathrm{cD}$ galaxies and powerful radio sources. Mon. Not. R. Astron. Soc. 268, 79-102 (1994).

5. Hughes, D. H., Dunlop, J. S. \& Rawlings, S. High-redshift radio galaxies and quasars at submillimetre wavelengths: assessing their evolutionary status. Mon. Not. R. Astron. Soc. 289, 766-782 (1997).

6. Archibald, E. N. et al. A submillimetre survey of the star-formation history of radio galaxies. Mon. Not. R. Astron. Soc. 323, 417-444 (2001).

7. Reuland, M., Röttgering, H. \& van Breugel, W. SCUBA observations of high redshift radio galaxies IN: 'Radio Galaxies: Past, present and future.' Elsevier Science, in press (astro-ph/0303321)

8. Holland, W. S. et al. SCUBA: a common-user submillimetre camera operating on the James Clerk Maxwell Telescope. Mon. Not. R. Astron. Soc. 303, 659-672 (1999).

9. Graham, A., Lauer T. R., Colless M., \& Postman M. Brightest cluster galaxy profile shapes. Astrophys. J. 465, 534-547 (1996).

10. Mihos, J. C., \& Hernquist, L. Gasdynamics and starbursts in major mergers. Astrophys. J. 464, 641-663 (1996).

11. Smail, I., Ivison, R. J. Blain, A. W. \& Kneib, J.-P. The nature of faint submillimetreselected galaxies. Mon. Not. R. Astron. Soc. 331, 495-520 (2002).

12. Scott, S. E. et al. The SCUBA 8-mJy survey - I. Submillimetre maps, sources and number counts. Mon. Not. R. Astron. Soc. 331, 817-838 (2002).

13. Chambers, K. C., Miley, G. K. \& van Breugel, W. Alignment of radio and optical orientations in high-redshift radio galaxies. Nature 329, 604-606 (1987).

14. McCarthy, P. J., van Breugel, W., Spinrad, H. \& Djorgovski, S. A correlation between the radio and optical morphologies of distant $3 \mathrm{CR}$ radio galaxies. Astrophys. J. 321, L29-L33 (1987).

15. Eales, S. A. A new theory for the alignment effect. Astrophys. J. 397, 49-54 (1992).

16. Smail, I. et al. A SCUBA galaxy in the protocluster around 52W002 at $z=2.4$. Astrophys. J. 583, 551-558 (2003).

17. Pentericci, L. et al. A Chandra study of X-ray sources in the field of the $\mathrm{z}=2.16$ radio galaxy MRC 1138-262 Astron. \& Astrophys. 396, 109-115 (2002).

18. Hildebrand, R. H. The determination of cloud masses and dust characteristics from submillimetre thermal emission. Qua. J. R. Astron. Soc. 24, 267-282 (1983).

19. Magorrian, J. et al. The demography of massive dark objects in galactic centers. Astron. J. 115, 2285-2305 (1998). 
20. Kauffmann, G. \& Haehnelt, M. A unified model for the evolution of galaxies and quasars. Mon. Not. R. Astron. Soc. 311, 576-588 (2000).

21. Page, M. J., Stevens, J. A., Mittaz, J. P. D. \& Carrera, F. J. Submillimetre evidence for the coeval growth of massive black holes and galaxy bulges. Science 294, 25162518 (2001).

22. Smail, I. et al. Chandra detections of SCUBA galaxies around High-z radio sources. Astrophys. J. submitted (2003).

23. De Breuck, C. et al. CO emission and associated HI absorption from a massive gas reservoir surrounding the $z=3$ radio galaxy B3 J2330+393. Astron. \& Astrophys. 401, 911-925 (2003).

24. Dunlop, J. S. et al. Quasars, their host galaxies, and their central black holes. Mon. Not. R. Astron. Soc. 340, 1095-1135 (2003).

25. Gorenstein, P. Empirical relation between interstellar X-ray absorption and optical extinction. Astrophys. J. 198, 95-101 (1975).

26. Ivison, R. J. et al. An excess of submillimeter sources near 4C 41.17: a candidate proto-cluster at $z=3.8$ ? Astrophys. J. 542, 27-34 (2000).

27. Omont, A. et al. Molecular gas and dust around a radio-quiet quasar at redshift 4.69. Nature 382, 428-431 (1996).

28. Papadopoulos, P. P. et al. $\mathrm{CO}(4-3)$ and dust emission in two powerful high- $z$ radio galaxies, and CO lines at high redshifts. Astrophys. J. 528, 626-636 (2000).

29. Percival, W. J., Scott, D., Peacock, J. A. \& Dunlop, J. S. The clustering of halo mergers. Mon. Not. R. Astron. Soc. 338, L31-L35 (2003).

30. Reuland, M. et al. An obscured radio galaxy at high redshift. Astrophys. J. 582, L71-L74 (2003).

\section{Acknowledgements.}

The JCMT is run by the Joint Astronomy Centre on behalf of the Particle Physics \& Astronomy Research Council (PPARC). JAS, JSD, IRS and WJP would like to acknowledge funding from PPARC, the Royal Society and the Leverhulme Trust. The work of WvB and MR was performed under the auspices of the U.S. Department of Energy, National Nuclear Security Administration by the University of California, Lawrence Livermore National Laboratory.

Correspondence and requests for materials should be addressed to Jason Stevens (jas@roe.ac.uk). 


\section{Tables.}

\section{Table 1. Target fields.}

\begin{tabular}{|c|c|c|c|c|c|c|c|c|}
\hline $\begin{array}{l}\text { Target } \\
\text { name }\end{array}$ & $\begin{array}{l}\text { Coordinates } a \\
\text { R.A. }\end{array}$ & $\begin{array}{l}(\mathrm{J} 2000) \\
\text { Dec. }\end{array}$ & $z$ & $S_{\text {peak }}^{b}$ & $S_{\text {total }}^{b}$ & $\begin{array}{l}\mathrm{M}_{\mathrm{d}}^{c} \\
\left(\mathrm{M}_{\odot}\right)\end{array}$ & $\begin{array}{l}\text { FWHM/P.A. } \\
\left(\begin{array}{l}d \\
\left({ }^{\circ}\right)\end{array}\right.\end{array}$ & $\begin{array}{l}\text { Radio size/P.A. } \\
\left({ }^{\prime \prime} /{ }^{\circ}\right)\end{array}$ \\
\hline $8 \mathrm{C} 1909+722$ & $1908 \quad 23.30$ & +722010.4 & 3.54 & $20.5 \pm 1.2$ & $34.9 \pm_{3.0}$ & $1.2 \times 10^{9}$ & $26 \times 9 @ 18$ & $14 @ 15$ \\
\hline 1. & $\begin{array}{lll}19 & 08 & 27.47\end{array}$ & +721928.0 & $\cdots$ & $17.6 \pm 1.2$ & $23.0 \pm 2.5$ & $\cdots$ & $12 \times 8 @ 169$ & $\cdots$ \\
\hline 2. & $\begin{array}{lll}19 & 08 & 29.31\end{array}$ & +722049.6 & $\cdots$ & $6.5 \pm 1.2$ & $8.7 \pm 2.4$ & $\cdots$ & $\cdots$ & $\cdots$ \\
\hline 3. & $\begin{array}{lll}19 & 08 & 16.12\end{array}$ & +722024.0 & $\cdots$ & $6.5 \pm 1.2$ & $4.3 \pm 2.1$ & $\cdots$ & $\cdots$ & $\cdots$ \\
\hline $8 \mathrm{C} 1435+635$ & 143637.33 & +63 1913.1 & 4.261 & $5.9 \pm 1.0$ & $6.0 \pm 2.1$ & $3.3 \times 10^{8}$ & $7 \times 0 @ 159$ & $4 @ 156$ \\
\hline 1. & 143632.46 & +632002.5 & $\cdots$ & $4.3 \pm 1.0$ & $3.9 \pm 1.8$ & $\cdots$ & $\cdots$ & $\cdots$ \\
\hline B3 J $2330+3927$ & 233024.91 & +392711.2 & 3.086 & $15.6 \pm 1.1$ & $22.2 \pm 2.7$ & $1.0 \times 10^{9}$ & $16 \times 7 @ 130$ & $2 @ 149$ \\
\hline 1. & $23 \quad 30 \quad 19.14$ & +392703.0 & $\cdots$ & $6.9 \pm 1.1$ & $8.2 \pm 1.9$ & $\cdots$ & $\cdots$ & $\cdots$ \\
\hline 2. & 233020.52 & +392657.7 & $\cdots$ & $5.0 \pm 1.1$ & $3.5 \pm 1.4$ & $\cdots$ & $\cdots$ & $\cdots$ \\
\hline PKS 1138-262 & 114048.25 & -262910.1 & 2.156 & $5.9 \pm 1.1$ & $6.7 \pm 2.4$ & $4.6 \times 10^{8}$ & $30 \times 0 @ 72$ & $11 @ 86$ \\
\hline 1. & 114053.38 & -262911.9 & $\sim_{2.16}$ & $9.9 \pm 1.1$ & $7.8 \pm 2.2$ & $\cdots$ & $17 \times 0 @ 60$ & $\cdots$ \\
\hline 2. & 114045.80 & -262956.6 & $\cdots$ & $5.9 \pm 1.1$ & $3.1 \pm 1.6$ & $\cdots$ & $\cdots$ & $\cdots$ \\
\hline 3. & 114045.61 & -262906.6 & $\cdots$ & $4.6 \pm 1.1$ & $2.2 \pm 1.4$ & $\cdots$ & $\cdots$ & $\cdots$ \\
\hline $4 \mathrm{C} 60.07$ & $05 \quad 1254.80$ & +603051.7 & 3.788 & $21.6 \pm 1.3$ & $23.8 \pm 3.5$ & $1.3 \times 10^{9}$ & $11 X 4 @ 110$ & $16 @ 107$ \\
\hline 1. & $05 \quad 1246.52$ & +603035.8 & $\cdots$ & $5.9 \pm 1.3$ & $6.3 \pm 2.1$ & $\cdots$ & $\cdots$ & $\cdots$ \\
\hline $4 \mathrm{C} 41.17$ & $0650 \quad 52.15$ & +413030.8 & 3.792 & $12.3 \pm 1.2$ & $12.0 \pm 2.3$ & $7.2 \times 10^{8}$ & $9 \times 6 @ 97$ & $20 @ 48$ \\
\hline 1. & $06 \quad 50 \quad 51.52$ & +413001.5 & $\cdots$ & $12.2 \pm 1.2$ & $21.2 \pm 2.9$ & $\cdots$ & $17 \times 15 @ 54$ & $\cdots$ \\
\hline 2. & $06 \quad 5049.25$ & +413001.5 & $\cdots$ & $7.1 \pm 1.2$ & $6.2 \pm 1.9$ & $\cdots$ & $8 \times 0 @ 50$ & $\cdots$ \\
\hline WN J0305+3525 & $\begin{array}{lll}03 & 05 & 47.42\end{array}$ & +352513.4 & $3 \pm 1$ & $12.2 \pm 1.0$ & $17.6 \pm 2.4$ & $6.9 \times 10^{8}$ & $19 \times 9 @ 9$ & $2 @ 64$ \\
\hline
\end{tabular}

a Coordinates of the HzRG companion sources are measured from the submillimetre maps. They are accurate to $4-5$ arcseconds.

${ }^{b} S_{\text {peak }}$ is the flux density per beam calculated at the peak of the dust emission. $S_{\text {total is the flux density }}$ of total dust emission calculated in an aperture. A calibration uncertainty of $\sim 10 \%$ is not included in these values. For some sources, the higher total flux densities reflect the extended nature of the dust emission, consistent with the finding that interferometric millimetre flux densities are often smaller than those calculated from single dish measurements. 28,23

${ }^{c}$ Dust mass calculated from the peak fluxes, and assuming a dust emissivity index, $\beta=2.0$; a dust temperature, $\mathrm{T}_{\mathrm{d}}=40 \mathrm{~K}$ typical of HzRGs; a mass absorption coefficient, $\mathrm{k}_{\mathrm{d}}(850 \mu \mathrm{m})=0.076 \mathrm{~m}^{2} \mathrm{~kg}^{-1}$; and an $\Omega_{\mathrm{m}}=0.3, \Omega_{\Lambda}=0.7, h=0.7$ cosmology. Our selection criteria result in a small spread in the calculated dust masses; an average value is $\sim 8 \times 10^{8} \mathrm{M}_{\odot}$. We can convert this dust mass into a conservative gas mass (molecular and atomic) assuming a standard Galactic gas-to-dust mass ratio of $200^{25}$ giving $1.6 \times 10^{11} \mathrm{M}_{\odot}$. We can confirm this estimate using the measured CO luminosities of three of the HzRGs, which can then be turned into molecular gas, and then total gas, mass using the standard Galactic conversion factor between $\mathrm{CO}$ luminosity and $\mathrm{H}_{2}$ mass and a ratio of atomic-to-molecular gas of $\sim 2$. In this manner, gas masses of $1.3-2.2 \times 10^{11} \mathrm{M}_{\odot}$ have been estimated for $4 \mathrm{C} 60.07,8 \mathrm{C} 1909+722$ and B3 J2330+3927. ${ }^{28,23}$ Hence the gas masses from the two methods are in reasonable agreement, and a representative estimate for the total gas mass in a HzRG with a submillimetre flux density of 10-15 mJy is thus $\simeq 2 \times 10^{11} \mathrm{M} \odot$. While this number is arguably uncertain by a factor of a few, it is very hard to argue that it can be inflated by an order of magnitude. ${ }^{5}$ Assuming an order of magnitude more mass is in 
the form of dark matter we estimate the halo masses to be $>10^{12} \mathrm{M}_{\odot}$.

${ }^{d}$ From a 2-dimensional Gaussian fit to the $850 \mu \mathrm{m}$ emission, deconvolved from the beam. All P.A.s are measured east of north. For the companion galaxies we give sizes for the brightest (highest S/N) sources only. A quoted size of zero arcseconds means that the source was not resolved in that direction.

${ }^{e}$ Quoted radio sizes are hotspot separations measured at $5 \mathrm{GHz}$.

${ }^{f}$ WNJ $0305+35$ lacks a robust spectroscopic redshift, but is thought to lie at $z=3 \pm 1$. $^{30}$ 


\section{Figure Captions.}

Figure 1. Continuum emission from dust in and around seven high-redshift radio galaxies. The data for 4C 41.17 have been published previously. ${ }^{26}$ Data were obtained using the SCUBA ${ }^{8}$ submillimetre camera on the James Clerk Maxwell Telescope (JCMT) in its dual-wavelength mapping mode during 1998-2001 in the top 20 percentile of Mauna Kea weather conditions. The beam, after smoothing to a FWHM of $14.4^{\prime \prime}$ at $850 \mu \mathrm{m}$, is shown bottom right in the form of a map of the blazar, 3C 345, with contours at -40 , $-30,-10,+10,+30,+50,+70,+90 \%$ of the central peak. The JCMT secondary mirror was chopped and nodded by $30^{\prime \prime}$ in Right Ascension (R.A.), resulting in the $-0.5 /+1.0 /-0.5$ beam profile. After all overheads, 30-40 ks were spent integrating on each of the fields, a total of $\sim 100 \mathrm{hr}$ before overheads, split down into 1280-s integrations separated by checks on focus, pointing accuracy and atmospheric opacity. All images have been cleaned with the smoothed beam. ${ }^{26}$ Submillimetre contours are shown at $-3,3,4$, $5,6,8,10 \times \sigma$, where $\sigma$ includes the contribution due to sources below the detection threshold and to the chopping and nodding procedure and ranges from 1 to $2 \mathrm{mJy}_{\text {beam }}{ }^{-1}$. The galaxy with the most compact appearance, $4 \mathrm{C} 41.17$, actually subtends $\sim 7.5^{\prime \prime}$ FWHM when deconvolved from the beam. The tick marks around the central radio galaxies show the direction of the kiloparsec scale radio jets.

Figure 2. Submillimetre extent of the radio galaxies. Radial profiles of the dust emission compared with that of the beam (solid line). The x-axis shows the radius from the centre of the radio galaxy dust emission (arcseconds) and the y-axis shows the normalised flux density. An accurate beam profile or point spread function (PSF) was measured with a $\sim 1 \mathrm{hr}$ mapping observation of the blazar 3C 345 which had an $850 \mu \mathrm{m}$ flux density of $>2 \mathrm{Jy}$ at the time. The image (shown in Figure 1) has a full width half maximum (FWHM) of 14.4". All subsequent size measurements are deconvolved from the PSF (see Table 1). The reality of the extended nature of the dust emission is established from observations of the quasar, BR 1202-0725, using the same technique and integration times used for the radio galaxies to mimic any systematic errors. BR $1202-0725$ serves as a particularly conservative comparison object - its dust continuum was resolved ${ }^{27}$ by the IRAM interferometer into two components separated by $4^{\prime \prime}$ (P.A. $\left.120 \pm 10^{\circ}\right)$. As expected, it appears relatively compact $\left(6.5^{\prime \prime} \times 5.5^{\prime \prime}\right.$ at P.A. $\left.6^{\circ}\right)$ in our submillimetre maps, and its radial profile is much more similar to that of the beam than to those of the HzRGs. We conclude that our observing procedure does not produce spurious morphologies, that the extended nature of the submillimetre emission from the radio galaxies is real and occurs on scales that are typically $\gg 4^{\prime \prime}$.

Figure 3. Observed alignment effects. The histograms show the offset in degrees between the kiloparsec scale radio structure of the HzRG and a) the position angle of the submillimetre source, b) the position angle defined by the projected vector joining the radio source to the brightest, spatially distinct submillimetre source, and c) the position angle defined by the projected vector joining the radio source to the second brightest, spatially distinct submillimetre source. In four cases these submillimetre sources are the companions listed in Table 1. In the remaining three cases one or both of the companions has a signal-to-noise ratio of 2 to 4; while these sources are not listed in Table 1 we use them in the analysis for completeness. We have applied the Kolmogorov Smirnov test to assess whether the apparent alignment effect seen in the first two of these plots is indeed statistically significant. Despite the small number statistics, we find that both of these apparent alignment effects are significant at the 2- $\sigma$ level (as compared to a random distribution; $p=0.04$ and $p=0.05$ respectively) whereas the corresponding result for the third distribution is not significant $(p=0.3)$.

Figure 4. Comparison with hierarchical models. The predicted distribution of number of companion sources per SCUBA field (after subtracting a background of 1 source per map for field contamination) from our $\Lambda-\mathrm{CDM}$ simulations shown against the observed distribution (shaded region). For the simulations we 
assume that the radio emission from the HzRG is powered by black holes with mass $\simeq 10^{9} \mathrm{M}_{\odot}{ }^{24}$, and therefore these lie at the heart of dark matter haloes with masses of $\simeq 10^{13} \mathrm{M}_{\odot}{ }^{19}$. Allowing for intrinsic scatter and uncertainties in this estimate, we therefore searched for virialized dark matter haloes with masses in the range $12.5<\log _{10}\left(M_{\text {halo }}\right) / \mathrm{M}_{\odot}<13.5$ at $z \simeq 3$ within an N-body simulation of a region of a $\Lambda-\mathrm{CDM}$ universe, covering a comoving volume of $\left(100 h^{-1} \mathrm{Mpc}\right)^{3}$. Parameters of the simulation were matched to the "concordance model" with $\Omega_{\mathrm{m}}=0.3, \Omega_{\Lambda}=0.7, h=0.7$; further details of the simulation are given elsewhere $\left(\Lambda \mathrm{CDM}_{100 a}\right)^{29}$. Within this simulation we identified 40 haloes which could be the counterparts of the HzRGs, and then investigated the mass distribution of other virialized dark-matter haloes within a sphere of projected diameter 3-arcmin (approximately the SCUBA field of view) around each of these. Within these regions we found typically 10 haloes with $M>10^{11} \mathrm{M}_{\odot}$, but on average only 1 halo with $M>10^{12} \mathrm{M}_{\odot}$. 


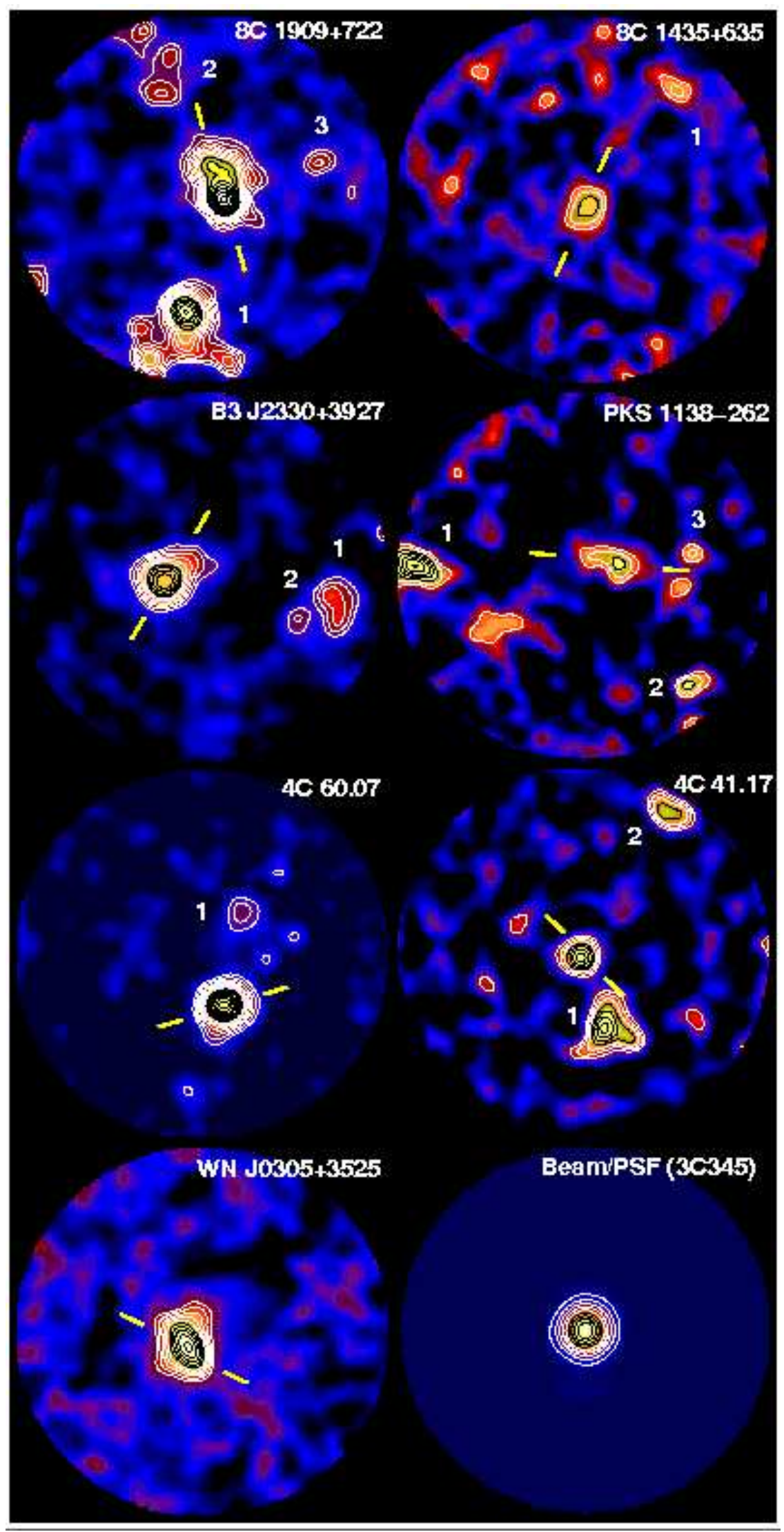



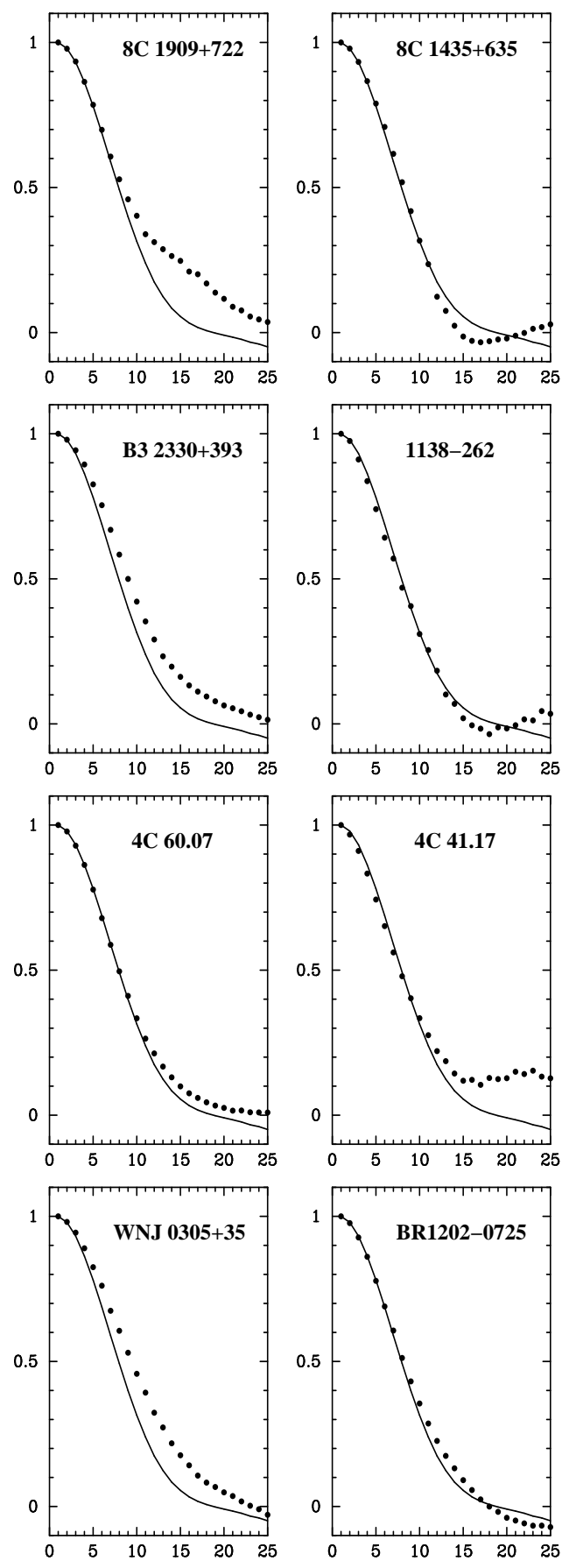

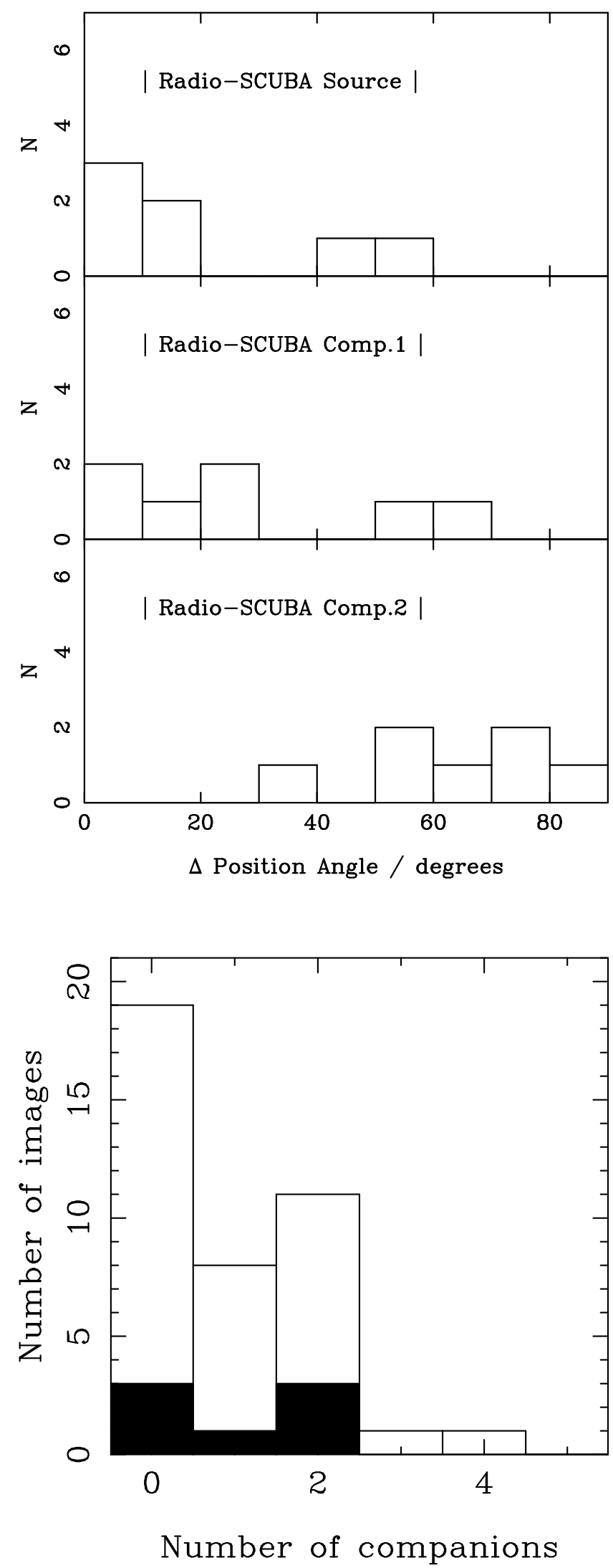